\title{
Lightpath routing considering differentiated physical layer constraints in transparent WDM networks
}

\author{
Lena Wosinska $^{\mathrm{a}^{*}}$, Amornrat Jirattigalachote ${ }^{\mathrm{a}}$, Paolo Monti ${ }^{\mathrm{a}}$, Anna Tzanakaki ${ }^{\mathrm{b}}$, Kostas Katrinis $^{\mathrm{b}}$ \\ ${ }^{a}$ Royal Institute of Technology KTH, School of Information and Communication Technology, \\ Isafjordsgatan 22, Electrum 229, 16440 Kista, Sweden \\ ${ }^{\mathrm{b}}$ Athens Information Technology (AIT), 19.5 Markopoulou Av., P.O. Box 68, 19002 Peania, Greece
}

\begin{abstract}
Wavelength division multiplexing (WDM) technology is considered to be the ultimate answer to the rapidly growing capacity demand of next generation networks. Many routing and wavelength assignment (RWA) algorithms, proposed for lightpath provisioning, base their routing decisions on the availability of network resources, and assume that optical fibers and components are ideal. In reality, physical impairments degrade the quality of the optical signal propagating through fiber segments and optical components. To cope with this problem, Impairment Constraint Based Routing (ICBR) algorithms, that consider physical impairments during connection provisioning, are currently proposed to prevent selecting lightpaths with poor signal quality. However, these algorithms support only a single (highest) quality of transmission threshold, the same one for all connection requests. This does not fit well with the variety of services, with potentially disparate QoS requirements, that the next generation networks are expected to support. Consequently, the efficiency of network resource utilization is reduced.

This paper demonstrates that a significant improvement, in terms of blocking probability, can be achieved when using an ICBR algorithm with differentiated physical layer constraints. Performance is compared with conventional impairment aware routing approaches when unprotected, shared path protected (SPP), and dedicated path protected (DPP) connection requests are considered. The achieved improvement is a result of more efficient resource utilization as unnecessary connection blocking can be avoided by selecting network resources offering optical signal quality that is "good enough" to satisfy a specific connection request.
\end{abstract}

OCIS codes: (060.0060) Fiber optics and optical communications; (060.4251) Networks, assignment and routing algorithms; (060.4257) Networks, network survivability; (060.4261) Networks, protection and restoration

\section{INTRODUCTION}

The latest advances in wavelength division multiplexing (WDM) technology make it possible to build all-optical transparent networks. These networks are considered to be the answer to the rapidly growing capacity demand of next generation networks and the future Internet. In transparent optical networks, the signal is transported from source to destination in the optical domain through all-optical channels (also referred to as lightpaths ${ }^{[1]}$ ), without the need for intermediate optoelectronic conversions. This results in significant cost saving in terms of both capital and operational expenditure. Another important advantage of all-optical networks is their transparency to bit rate and traffic format.

WDM networks are able to transport hundreds of wavelength channels through a single optical fiber, with a capacity of $10 \mathrm{Gbit} / \mathrm{s}, 40 \mathrm{Gbit} / \mathrm{s}$ or even $100 \mathrm{Gbit} / \mathrm{s}$ per channel ${ }^{[2]}$. One single fiber-cable may also consist of a large number of optical fibers. Consequently, a single fiber-cable cut may lead to the interruption of a huge number of optical connections and the loss of an enormous amount of data. In order to avoid service interruption and loss of data, it is extremely important to provide an efficient survivability mechanism in all-optical network. WDM networks can be made survivable by means of protection switching schemes implemented in the WDM layer ${ }^{[3]}$. A protection scheme requires the allocation of spare (or standby) resources that can be used in the event of a fault occurrence. For a lightpath, the protection scheme consists of assigning a working and a protection path between the source and the destination. The working path carries the

\footnotetext{
* email: wosinska@kth.se, phone: +46 8790 4242, fax: +46 8790 4090, web: http://www.ict.kth.se/MAP/FMI/Negonet/.
} 
offered traffic during normal network operations. When the working path fails, the interrupted traffic is rerouted over the protection path.

Each working (and protection) lightpath created in a WDM network is assigned both a route and a wavelength (assuming that wavelength conversion is not an option, i.e., the wavelength continuity constraint is enforced). This is the so-called (conventional) routing and wavelength assignment (RWA) problem. One of the RWA problem objectives is to reserve the minimum number of network resources (wavelengths) for all traffic demands. It is expected that by minimizing the amount of reserved resources, the blocking probability is reduced - where a demand is blocked in case it cannot be established because of lack of available resources in the network.

The RWA problem is known to be NP complete. Many RWA algorithms, proposed in the literature to solve this problem ${ }^{[3][4][5][6]}$, assume that the optical layer (i.e., fibers and components) is an ideal medium and that each solution (i.e., lightpath) is valid and feasible. However, in a transparent optical network physical impairments, inherent to optical transmission, degrade the quality of the optical signal propagating through fiber segments and optical components ${ }^{[7]}$. The physical impairments present in an optical network can be divided into linear and non-linear. Linear impairments (Amplified Spontaneous Emission (ASE) noise, Group Velocity Dispersion (GVD) and Polarization Mode Dispersion (PMD), just to name a few) do not depend on the signal power and affect each channel individually. Non-linear impairments (e.g., Self Phase Modulation (SPM), Cross Phase Modulation (XPM) and Four Wave Mixing (FWM)) on the other hand, affect each channel individually but their effect can impact the performance of channels as well ${ }^{[8][9]}$.

The effect of optical impairments on the quality of the transmission is even stronger in survivable WDM networks ${ }^{[10][11][12]}$. In fact the protection paths are typically much longer than the primary paths and, consequently, more susceptible to physical layer impairments. This in turn degrades the quality of the signal traversing the protection paths and has also a direct impact on the working path, since the working-protection path-pair might not be able to provide the required signal quality ${ }^{[12]}$. In order to guarantee sufficient quality level of transmission in an efficient way, optical physical impairments need to be considered as additional constraints while solving the RWA problem.

In other words, in the presence of a non-ideal optical transmission medium, linear and non-linear physical impairments play a key role in solving the so-called Impairment Aware (IA)-RWA problem. The objective of the IA-RWA problem is not only to reserve the minimum of network resources, but also to guarantee the required quality level of transmission, e.g., measured in terms of bit error rate (BER), for each connection. Several Impairment Constraint Based Routing (ICBR) algorithms have been proposed in the literature to solve the IA-RWA problem for both non-survivable ${ }^{[13][14]}$ and survivable $\mathrm{e}^{[10][11][15]} \mathrm{WDM}$ networks ${ }^{1}$. Usually they are referred to as Impairment-Aware Best-Path (IABP) routing algorithms, since they always assign each connection the least impaired path, regardless of the signal quality requirement. However, next generation networks and the future Internet are expected to support a variety of services, e.g., peer-to-peer (P2P) applications, high definition television (HDTV), and Audio Video On Demand (AVOD), with potentially disparate QoS requirements, including different, and service-based, quality of transmission thresholds.

Despite this variety of requirements - e.g., quality of transmission, bandwidth, and delay - state of the art ICBR schemes ${ }^{[10][11][13][14][15]}$ treat all connection requests in a flat manner. They support only a single quality of transmission threshold, the same one for all connection requests (the lowest BER value, in case of IABP routing) and are unable to differentiate among the specific applications requesting the connection provisioning. As a consequence, such singlethreshold approaches may (i) unnecessarily block connection requests that otherwise could sustain higher BER threshold and (ii) for those connection requests that are accepted, a single-threshold scheme may overprovision network resources, with a detrimental effect on the overall blocking probability. To overcome these deficiencies, the authors recently proposed an ICBR algorithm, referred to as ICBR-Diff ${ }^{[16][17]}$ supporting differentiation of services at the BER level. In the proposed approach, various BER thresholds are considered for accepting/blocking connection requests at the connection provisioning phase, depending on QoS requirements.

This paper presents comprehensive evaluation, in terms of blocking probability, of the ICBR-Diff algorithm applied to both protected and unprotected connection requests, based on both shared path protection (SPP) and dedicated path protection (DPP). The obtained results reveal that by adapting the lightpath provisioning phase to the connection BER requirements, the algorithm provides the best quality lightpaths only when required, and in this way over provisioning of

\footnotetext{
${ }^{1}$ In the latter case, ICBR solutions that jointly address resilience and physical layer performance requirements are preferred [15] over the conventional routing approaches.
} 
network resources is avoided. Consequently, a significant blocking probability reduction can be achieved when compared to shortest path and IABP approaches.

\section{SERVICE DIFFERENTIATION APPROACH}

In this section, an ICBR algorithm capable of supporting service differentiation based on the transmission quality level (i.e., BER) of each connection request is presented.

\subsection{Definitions and assumptions}

It is assumed that the bandwidth demand of each connection request is one wavelength unit and that wavelength conversion capability is not available, i.e. wavelength continuity constraint is enforced while solving the routing problem. As mentioned in Section 1, ICBR-Diff supports differentiation of services ${ }^{[16]}$, whereby connection requests are divided into two distinct classes with regards to their signal quality requirements, i.e. Class-1 connection requests that require higher signal quality in terms of maximum tolerated BER, and Class- 2 connection requests that can tolerate, higher signal degradation than Class-1. In ICBR-Diff, the path selection procedure chooses the path with maximum BER from a set of $k$ feasible paths. This is different from traditional impairment-aware best-path (IABP) routing, where the path with minimum BER is always chosen.

In this work, the effect of physical impairments is quantified by using the quality factor Q, based on the model presented $\mathrm{in}^{[14]}$. The Q-factor under consideration includes both linear and nonlinear physical impairments, namely Amplified Spontaneous Emission (ASE) noise, Four-Wave Mixing (FWM), the combined Self-Phase Modulation/Group Velocity Dispersion (SPM/GVD) and optical filtering effects, and Cross-Phase Modulation (XPM). ASE, FWM and XPM are calculated assuming a Gaussian distribution. For the combined SPM/GVD and optical filtering effects, they are measured using an eye closure penalty metric calculated on the most degraded bit-pattern.

\subsection{An impairment constraint based routing algorithm with service differentiation (ICBR-Diff)}

In Figure 1, the flow chart of the ICBR-Diff algorithm is presented. The algorithm starts with an initialization phase where the network topology information is collected, e.g. number of nodes, number of links, link lengths, link capacities, and all the physical parameters required for the calculation of the Q-penalty of each link. The rest of the algorithm consists of two phases: primary path provisioning and protection path provisioning (both dedicated and shared path protection is supported).

In the primary path provisioning phase there are two routing algorithms, shortest path and impairment constraint routing. The cost of each link is assigned according to the chosen routing algorithm. In the case of the shortest path approach, the link length is used as link cost, while the Q-penalty is considered as link cost for the impairment constraint approach. In addition, the cost of a link is set to infinity if all wavelengths on that particular link are occupied by already provisioned lightpaths. After assigning link costs, the $k$ alternative routes for each connection request are computed using the Dijkstra algorithm. If there is at least one common wavelength available on every link, the route is stored in the set of candidate routes. Otherwise, if no route is found or there is not a common wavelength, the connection request is blocked. Next, the BER of each candidate route is calculated. In the case of shortest path routing, the shortest route in the set of candidate routes is selected first, and then the BER of this route is calculated. In the cases of traditional IABP routing and the ICBR-Diff algorithm, the BER of all candidate routes is calculated. With shortest path routing, if the BER of the selected shortest route is higher than a pre-defined signal quality threshold, i.e. one single BER threshold for all the connection requests independently of their class, the respective connection request is blocked. In the case of the ICBR-Diff algorithm, the BER of each candidate route is compared against the signal quality requirement of the connection request, i.e. different BER thresholds according to the class of the connection requests, and the route with maximum BER that satisfies the signal quality requirement ("good enough") is selected. In contrast, in the case of the traditional IABP algorithm the route with minimum BER is selected and compared against the single BER threshold. In both traditional IABP routing and with the ICBR-Diff algorithm, if none of the candidate routes satisfies the signal quality threshold, the connection request is blocked. Next, the first wavelength in the list of available wavelengths for the selected route is chosen (First-Fit) and assigned to the lightpath for the particular connection request.

In the protection path provisioning phase, the links used in the primary path are not considered in the topology while computing the protection path. This will result in a path that is link-disjoint from the primary path. Then, link costs are assigned. In the case of dedicated path protection (DPP), the link cost assignment procedure works in the same way as in the primary path link cost assignment. In the case of shared path protection (SPP), link costs are set to zero for all those 
wavelengths that are sharable, i.e., the wavelength used by already provisioned protection paths whose primary paths are link-disjoint from the one under consideration. Link costs for other wavelengths (i.e., the ones that are not sharable) are assigned in the same way as in the primary path link cost assignment. For both protection schemes, i.e. DPP and SPP, BER checking and wavelength assignment are carried out in the same way as in the primary path provisioning phase.

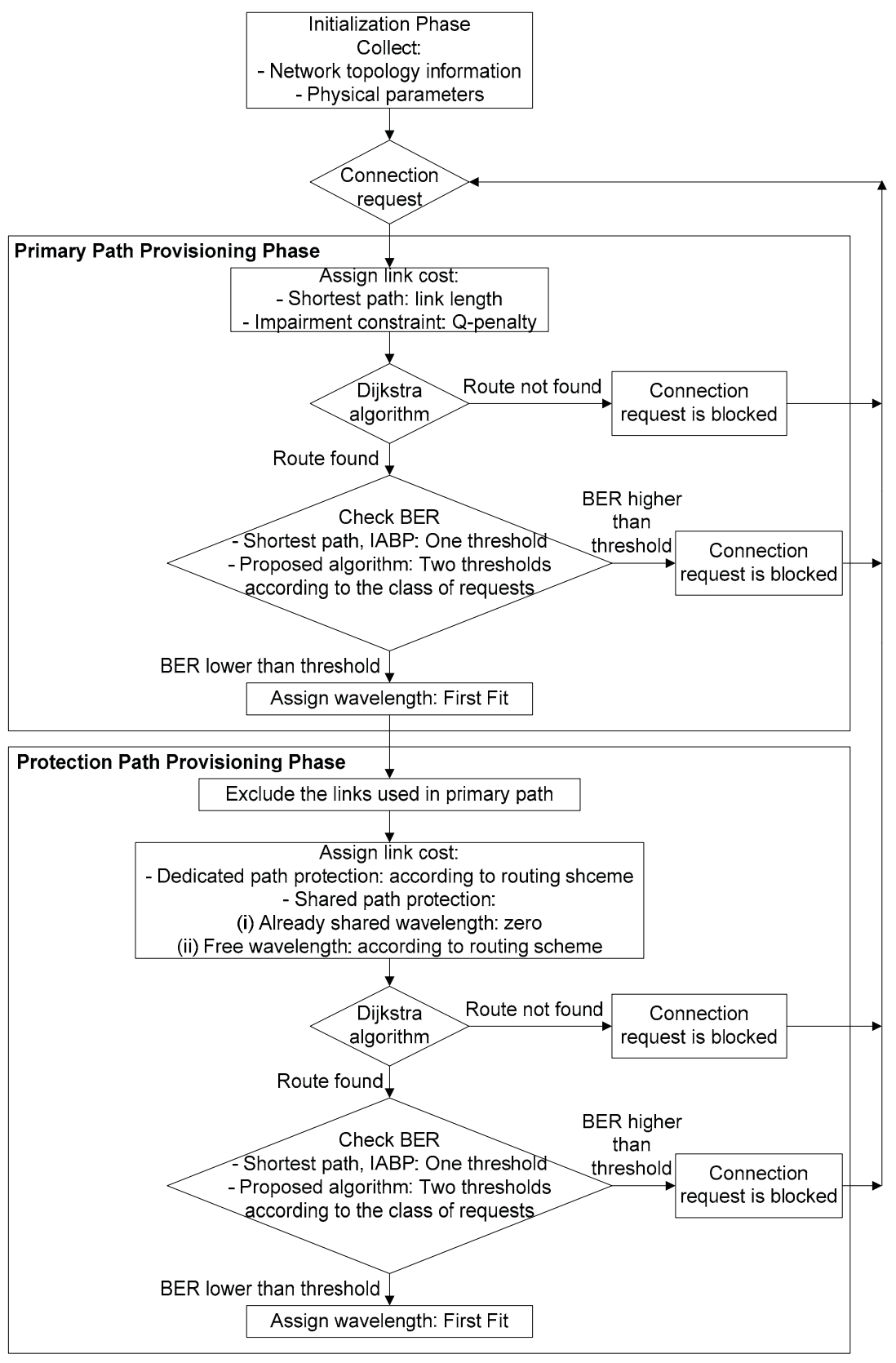

Figure 1. The impairment constraint based routing algorithm with service differentiation (ICBR-Diff). 


\section{RESULTS}

The ICBR-Diff algorithm is evaluated through simulations, using the Pan-European network topology (Figure 2) defined by COST $239^{[18]}$. The topology consists of 11 nodes and 26 bidirectional fiber links with 16 wavelengths per fiber. In order to differentiate between two distinct classes of connection requests we used signal quality requirements quantified via BER. The signal quality requirements of Class- 1 and Class- 2 connection requests were chosen based on available IP traffic measurements ${ }^{[19][20]}$. It is shown that streaming media traffic, i.e. traffic requiring higher signal quality (Class-1), accounts for much less of the total bandwidth utilization, whereas peer-to-peer and World Wide Web (WWW) traffic, i.e. traffic requiring lower signal quality (Class-2), is dominating.

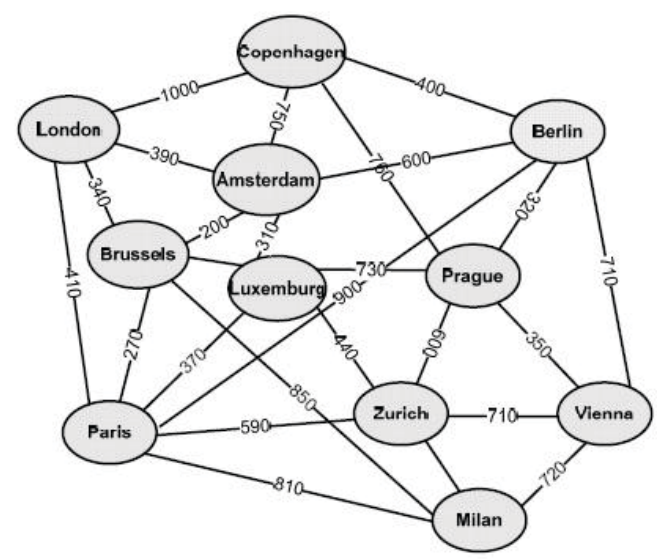

Figure 2. Pan European network topology.

In our simulations, Class-1 and Class-2 connection requests are assumed to require BER less than $10^{-15}$ and $10^{-9}$ respectively. According to the ICBR-Diff algorithm, Class-1 connection request is blocked if there is no available lightpath connecting source and destination that exhibits BER less than $10^{-15}$. A connection request of Class- 2 is blocked if no lightpath is able to provide a BER value less than $10^{-9}$. For benchmarking purposes, we also evaluate two additional provisioning algorithms, namely shortest path (SP) and IABP. Essentially these two approaches do not employ service differentiation, but instead handle both Class-1 and Class-2 with a single transmission quality threshold, i.e., they block all the connection requests (regardless of the service class) with BER greater than $10^{-15}$. In our experimental model, we assume Poisson arrival of connection requests and uniform distribution of source/destination node pairs. Furthermore, the lifetime of each connection request is exponentially distributed and specified at the provisioning instant. In our experiment we assumed that the traffic comprises of 30\% of Class- 1 connection requests and $70 \%$ of Class- 2 connection requests. For each chosen network load value, the blocking probability is recorded after the steady state is detected (transient interval is removed) and we execute enough number of repetitions until the $90 \%$ confidence interval of measured mean blocking probability becomes less than $10 \%$ relative to the measured mean.

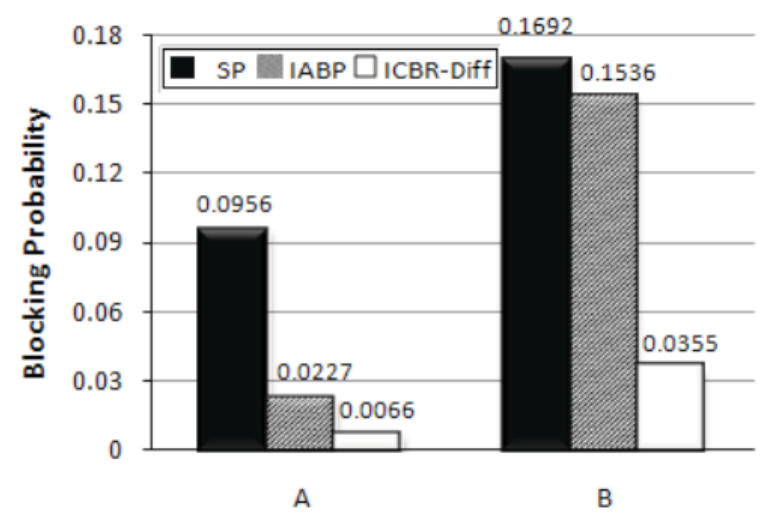

Figure 3. Protection is not required. Blocking probability due to insufficient signal quality as a function of the network load. Two cases are considered, A: 60 Erlangs (low load) and B: 300 Erlangs (high load). 
Figure 3 compares the blocking probability due to insufficient signal quality when SP, IABP and ICBR-Diff is applied to the connection requests that do not require any protection. At low load (60 Erlangs, case A) there are sufficient resources in the network and, consequently, connection requests are blocked only because of the violation of signal quality requirement due to physical impairments. On the other hand, at high load (300 Erlangs, case B) connection requests are blocked due to both insufficient network resources and physical impairments. We show the blocking probability due to insufficient signal quality to emphasize the difference in treating impairments by SP, IABP and ICBR-Diff. In low load conditions (case A) IABP reduces the influence of physical layer impact on blocking when compared to SP and further significant improvement can be obtained when using ICBR-Diff. In high load conditions (case B) on the other hand the improvement yield by IABP compared to SP is not very high. This is because low impaired paths are assigned to connection requests regardless of the class the connection belongs to. This in turn reduces the probability of finding high quality paths for connection requests of Class-1. In this case the differentiated use of network resources proposed by ICBR-Diff allows for a drastic improvement of the blocking performance.

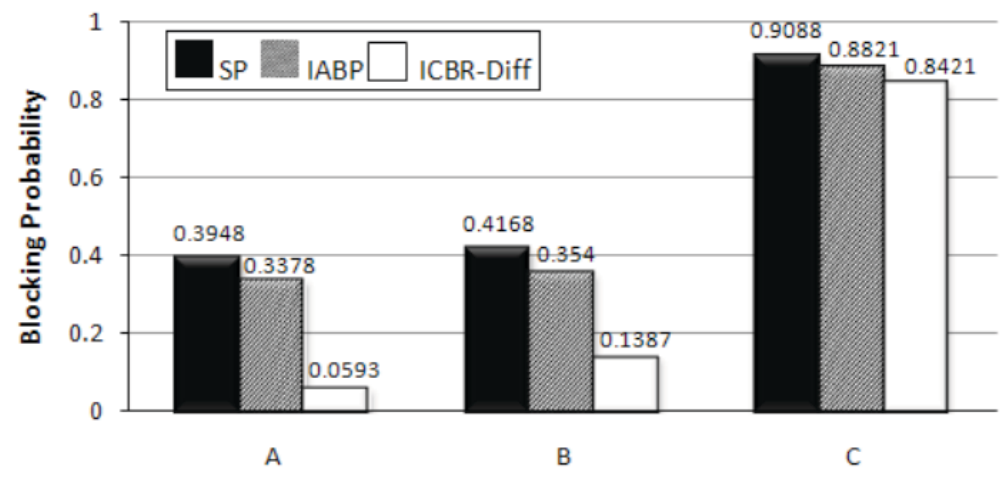

Figure 4. Dedicated path protection (DPP). Case A: blocking probability (due to insufficient signal quality) in low load conditions (60 Erlangs). Case B: blocking probability (due to insufficient signal quality) in high load conditions (300 Erlangs). Case C: total blocking probability (due to insufficient signal quality and insufficient network resources) in high load conditions (300 Erlangs).

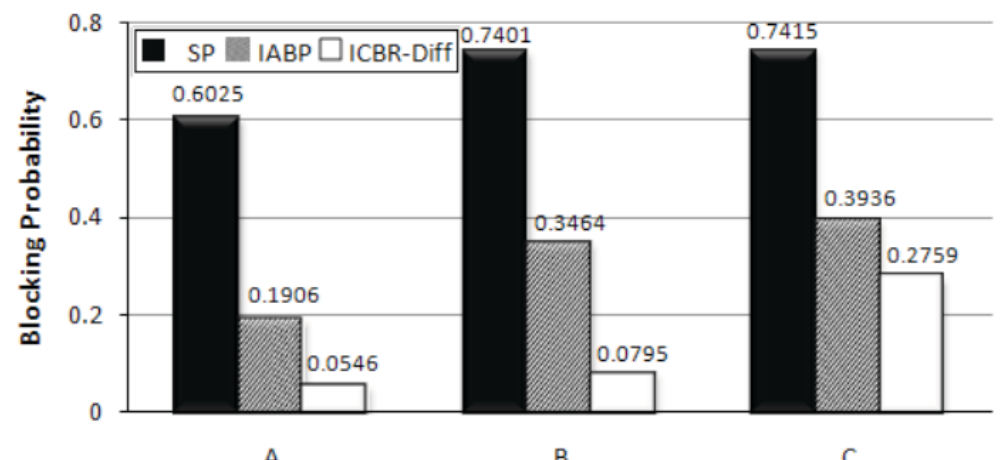

Figure 5. Shared path protection (SPP). Case A: blocking probability (due to insufficient signal quality) in low load conditions (60 Erlangs). Case B: blocking probability (due to insufficient signal quality) in high load conditions (300 Erlangs). Case C: total blocking probability (due to insufficient signal quality and insufficient network resources) in high load conditions (300 Erlangs).

Figure 4 and Figure 5 compare the blocking probability performance of SP, IABP and ICBR-Diff when all connection requests require dedicated path protection (DPP) and shared path protection (SPP), respectively. Great improvement of the network blocking probability can be achieved when impairment aware routing is applied to both the primary and the protection path, when compared to SP routing. The figures also show how ICBR-Diff is able to outperform IABP due to its inherent improved utilization of high quality links. It can be noticed that under high loading conditions, blocking due to insufficient resources (case C) is dominating in the DPP scheme. On the other hand, when using the SPP scheme 
blocking probability due to violation of signal quality requirement plays the most important role when SP and IABP algorithms are used (compare case B and case C in Figure 4 and Figure 5 respectively). This is because in SPP protection paths tend to share resources as much as possible, which makes them longer than the protection paths in the case of DPP. This has a negative effect on the signal quality of protection paths in SSP because longer paths are typically more impaired. As expected, both figures show that the total blocking probability is higher for DPP than SPP.

\section{CONCLUSIONS}

In this paper it is demonstrated that a significant improvement, in terms of blocking probability, can be achieved by impairment constraint based routing (ICBR) algorithm with differentiated physical layer constraint. Performance results are compared with conventional shortest path and Impairment Aware Best Path (IABP) routing approaches. The blocking probability improvement is the result of more efficient resource utilization where unnecessary connection blocking can be avoided by selecting network resources offering optical signal quality that is "good enough" for a specific connection request.

\section{ACKNOWLEDGEMENTS}

This work was supported by the Network of Excellence "Building the Future Optical Network in Europe" (BONE), funded by the European Commission through the 7th ICT-Framework Programme and the EUREKA/CELTIC project "Management Platform for Next Generation Optical Networks" (MANGO).

\section{REFERENCES}

[1] I. Chlamtac, A. Ganz, and G. Karmi, "Lightpath communications: a novel approach to high bandwidth optical WANs," IEEE Transactions on Communications, vol. 40, no. 7, pp. 1171-1182, 1992.

[2] J. Berthold, A.A.M. Saleh, L. Blair, J.M. Simmons, "Optical networking: past present and future", Journal of Lightwave Technology, vol. 26, no. 9, pp. 1104-1118, 2008.

[3] "Special issue on protection and survivability in optical network," Optical Network Magazine, vol. 2, no.4, July/August, 2001.

[4] H. Zang, J.P. Jue, B. Mukherjee, "A review of routing and wavelength assignment approaches for wavelengthrouted optical WDM netwroks", Optical Network Magazine, vol. 1, no 1, pp. 47-59, 2000.

[5] R. Ramaswami and K. N. Sivarajan, "Routing and wavelength assignment in all-optical networks", IEEE/ACM Transaction on Networking, vol. 3, no. 5, pp. 489-500, Oct. 1995.

[6] D. Cavendish, A. Kolarov and B. Sengupta, "Routing and wavelength assignment in WDM mesh networks", in Proc. Conf. Globecome 2004, Dec. 2004.

[7] J. Strand, A. L. Chiu and R. Tkach, "Issues for routing in the optical layer", IEEE Communications Mag., vol. 39, no. 2, pp. 81-87, Feb. 2001.

[8] M. Farahmand, D. Awduche, S. Tibuleac, D. Atlas, "Characterization and representation of impairments for routing and path control in all-optical networks", in Proc. Of Nat. Fiber Optic Engineers Conference (NFOEC), Dallas, TX, Sept. 2002.

[9] B. Ramamurthy, D. Datta, H. Feng, J.P. Heritage, B. Mukherjee, "Impact of transmission impairments on the teletraffic performance of wavelength-routed optical networks", Journal of Lightwave Technology, vol.10, no. 17, pp. 1713-1723.

[10] Y. Zhai, Y. Pointurier, S. Subramaniam, M. Brandt-Pearce, "Performance of dedicated path protection in transmission impaired DWDM networks", in Proc. IEEE ICC, pp. 2342-2347, Glasgow, UK, June 2007.

[11] X. Yang, L. Shen, B. Ramamurthy, "Survivable lightpath provisioning in WDM mesh networks under shared path protection and signal quality constraints", Journal of Lightwave Technology, vol. 23, no. 4, pp. 1156-1567, 2005.

[12] G. Markidis and A. Tzanakaki, "Routing and wavelength assignment algorithms in survivable WDM networks under physical layer constraints", in Proceedings of the 3rd International GOPS Workshop, IEEE Broadnets, 2008.

[13] Y. Huang, J. P. Heritage and B. Mukherjee, "Connection provisioning with transmission impairment consideration in optical WDM networks with high-speed channels", Journal of Lightwave Technology, vol. 23, no. 3, pp. 982993, Mar. 2005.

[14] G. Markidis, S. Sygletos, A. Tzanakaki and I. Tomkos, "Impairment aware based routing and wavelength assignment in transparent long haul networks", in Proc. Conf. on Optical Network Design and Modeling (ONDM), May 2007. 
[15] A. Tzanakaki, K. Georgakilas, K. Katrinis, L. Wosinska, A. Jirattigalachote and P. Monti, "Network performance improvement in survivable WDM networks considering physical layer constraints", in Proc. of IEEE ICTON/RONEXT'09, June 2009.

[16] A. Jirattigalachote, K. Katrinis, A. Tzanakaki, L. Wosinska and P. Monti, "Quantifying the benefit of BER-based Differentiated Path Provisioning in WDM Optical Networks", in Proc. of IEEE ICTON/RONEXT'09, June 2009.

[17] A. Jirattigalachote, L. Wosinska, P. Monti, K. Katrinis, and A. Tzanakaki, "Impairment Constraint Based Routing (ICBR) with Service Differentiation in Survivable WDM Networks", in Proc. of ECOC2009, September 2009.

[18] P. Batchelor et al., "Study on the implementation of optical transparent transport networks in the European environment-Results of the research project COST 239", Photonic Network Communications, vol. 2, no. 1, pp. 1532, 2000.

[19] Traffic Measurements and Models in Multi-Service Networks project, Celtic project, "TRAMMS IP Traffic report no. 1, April 2008", http://projects.celtic-initiative.org/tramms/.

[20] Traffic Measurements and Models in Multi-Service Networks project, Celtic project, "TRAMMS IP Traffic report no. 3, June 2008", http://projects.celtic-initiative.org/tramms/. 